\title{
Developing Personalized Intelligent Interior Units to Promote Activity and Customized Healthcare for Aging Society
}

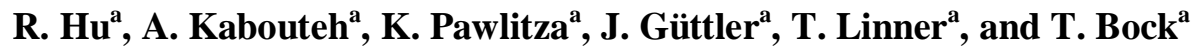 \\ ${ }^{a}$ Chair of Building Realization and Robotics, Technical University of Munich, Germany \\ E-mail: rongbo.hu@br2.ar.tum.de, amir.kabouteh@br2.ar.tum.de, katja.pawlitza@ br2.ar.tum.de, \\ joerg.guettler@br2.ar.tum.de, thomas.linner@br2.ar.tum.de, thomas.bock@br2.ar.tum.de
}

\begin{abstract}
-
The world's population is aging at an unprecedented pace. Aging society is not only a severe crisis in the developed world such as Germany and Japan, but also a rigorous challenge in emerging economies such as China. Many agerelated diseases are fostered by the lack of physical, cognitive, and social activities. Increasing the activity level has many benefits for the elderly and can improve their independence. The research project REACH (funded by European Union's Horizon 2020 Research and Innovation Program under grant agreement No. 690425) aims to develop a service system that will turn clinical and care environments into personalized modular sensing, prevention, and intervention systems that encourage the elderly to become healthy through activities (e.g., physical, cognitive, socializing, personalized food, etc.). As a core research partner, the Chair of Building Realization and Robotics at Technical University of Munich developed a series of Personalized Intelligent Interior Units (PI $\left.{ }^{2} \mathrm{Us}\right)$, which are a special type of smart furniture, that materialize the REACH concepts and functionality seamlessly into the different REACH use case settings. Specifically, the design and functions of the $\mathrm{PI}^{2} \mathrm{U}$, which consist of the PI'U-SilverArc, $\mathrm{PI}^{2} \mathrm{U}$-MiniArc, $\mathrm{PI}^{2} \mathrm{U}$-Bed, and $\mathrm{PI}^{2} \mathrm{U}$-iStander, will be described in detail. In addition, a modular apartment integrating all $\mathrm{PI}^{2} \mathrm{Us}$ and key technologies in REACH is designed and simulated to create a total interior living and care environment for elderly users. Due to its modularity, parts of the apartment can be easily adapted and rapidly deployed in different REACH use case settings in four European countries, which will then help the REACH consortium execute a series of testing activities. In conclusion, this research project provides a systematic and innovative example for the world to mitigate the impact of aging society.
\end{abstract}

Keywords -

Aging society; Assistive technology; Personalized Intelligent Interior Units; Physical and cognitive activities; Sensing technology

\section{Background}

The world's population is aging at an unforeseen rate. Aging society is a crisis that is experienced all around the globe, including developed countries such as Germany and Japan, and emerging economies such as China. For instance, the health expenditure in the EU is expected to rise by $350 \%$ by 2050 compared to an economic growth of only $180 \%$ in the same timeframe [1]. The Directorate General for Economic and Financial Affairs reported that the provision of longterm care (LTC) in particular will pose an increasing challenge to the sustainability of public finances in the $\mathrm{EU}$, due to an aging population [2]. Evidently, many age-related diseases are fostered by a lack of physical, cognitive, and social activities. Increasing the level of activity has many benefits for the elderly and can improve their level of independence [3]. Therefore, the project REACH represents a solution that seeks to protect elderly citizens against loss of function and a decline in ability to independently perform Activities of Daily Living (ADLs), which ultimately lead to entering LTC. REACH will allow European industries, including small- and medium-sized enterprises, to capitalize on the European high-tech knowhow, make Europe a world leader in prevention technologies, services, and underlying healthcare ICT platforms, and meanwhile tackle the ultimate crisis of rising healthcare expenditures.

\section{Introduction to REACH}

REACH stands for Responsive Engagement of the Elderly promoting Activity and Customized Healthcare. 
With a project duration of four years, it is a crossdisciplinary, multi-partner, EU-funded research project that develops the future generation of technologies and services for a value-based and prevention-oriented health care system. The key elements and concepts of $\mathrm{REACH}$ will be explained as follows.

\subsection{Objectives and the consortium}

In the project $\mathrm{REACH}$, an experimental, crossdisciplinary sensing-monitoring-intervention system is developed that can be placed in an unobtrusive way in various care settings and living environments of elderly citizens. The system aims at 1) using a variety of sensors to detect specific vital signs, behavioral patterns, and health status; 2) predicting future health status, risks or events; and 3) anticipatorily providing a series of customized health products and services that support and promote physical activities. As a result, the Healthy Life Years (HLY) of the elderly would be increased, and their time spent in LTC facilities would be reduced. The REACH consortium consists of six research partners who serve as the scientific backbone of the project; seven industry partners who undertake various tasks from sensor and rehabilitation device development, to ICT platform integration, standardization support, and business model generation; and four application partners who take on the real-world deployment of the technologies developed in $\mathrm{REACH}$ in their exemplary care environments [4].

\subsection{Touchpoints and Engine concept}

As a major achievement of the first project year, the REACH consortium developed the "Touchpoints and Engine" concept as a comprehensive solution of REACH's system architecture. It further guides the detailed structural relation between the subsystems of REACH. With the "Touchpoints and Engine" concept, REACH's product-service-system architecture is divided into six manageable research and development clusters: four Touchpoint (TP) clusters (in other words, work groups each focusing on a specific topic within the project, including TP1: Personal Mobility Device, TP2: Active Environment, TP3: Socializing \& Nutritional Monitoring/Intervention, and TP4: Gaming \& Training System) that represent tangible connections between users (e.g., seniors, caregivers, physicians, etc.) and the REACH system; one "Engine" cluster which is a cloudbased digital platform serving as the brain of the project; and one "Interface" cluster which is composed of a set of means that allow the TPs and other products and services to interact with the Engine. Each cluster is associated with a dedicated and independent development team coming from the project consortium members (see Figure 1).

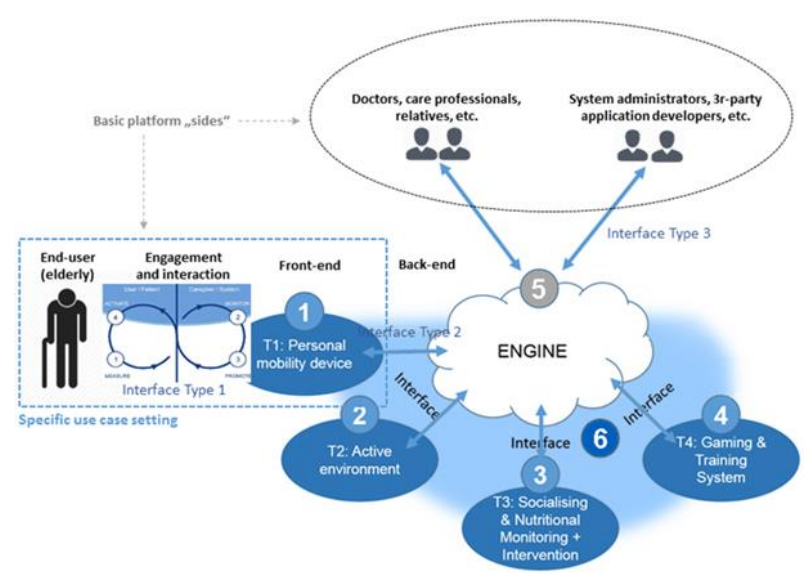

Figure 1. "Touchpoints and Engine" concept in $\mathrm{REACH}$

\section{Personalized Intelligent Interior Units}

The Personalized Intelligent Interior Unit $\left(\mathrm{PI}^{2} \mathrm{U}\right)$ is a special type of smart furniture that seamlessly integrates the REACH concepts and functionality into the different REACH use case settings. This paper focuses on a series of $\mathrm{PI}^{2} \mathrm{Us}$ developed by the project team in TP2 (i.e., the "Active Environment" work group of the project). The relevant $\mathrm{PI}^{2} \mathrm{Us}$ include $\mathrm{PI}^{2} \mathrm{U}-\mathrm{Silver} A r c$, $\mathrm{PI}^{2} \mathrm{U}-$ MiniArc, $\mathrm{PI}^{2} \mathrm{U}-\mathrm{Bed}$, and $\mathrm{PI}^{2} \mathrm{U}-\mathrm{i}$ Stander.

\subsection{PI ${ }^{2} U-S i l v e r A r c$}

The PI ${ }^{2} U-S i l v e r A r c$ was developed for the use in a large kitchen or dining space (e.g., a community kitchen). It offers an interactive projection area in the kitchen, where recipes and games can be displayed. It also has a foldaway projection area where a training program can be displayed (see Figure 2). The round shapes, wood material, and bright colors give the $\mathrm{PI}^{2} \mathrm{U}$ SilverArc a warm and inviting appearance.

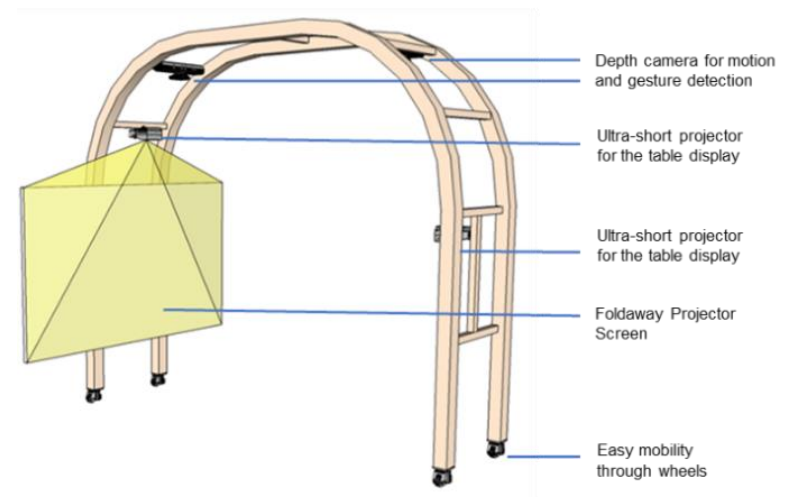

Figure 2. Preliminary design of the $\mathrm{PI}^{2} \mathrm{U}-$ SilverArc 
The design of the $\mathrm{PI}^{2} \mathrm{U}-$ SilverArc cannot be directly converted into a prototype. For example, the material of the prototype and the final product differ substantially. After evaluation, it was decided that MayTec's modular aluminum profile system is suitable for building the prototypes (maytec.com.de). MayTec offers a wide range of modular profiles, accessories, and different connection possibilities. Simple changes can readily be made after the first test by using the aluminum profile system. Furthermore, in order to lower the costs, the curves were omitted in the prototypes because they mainly serve an aesthetic function and are not necessary for the testing.

Figure 3 shows in detail how the technical equipment is integrated into the prototype. An ultrashort projector is fixed above the projection screen. It was purposely decided against a mounting under the projection area, since the dust load for the projection lens would increase. Depth cameras are attached to a sliding system so that their positioning can be adjusted.

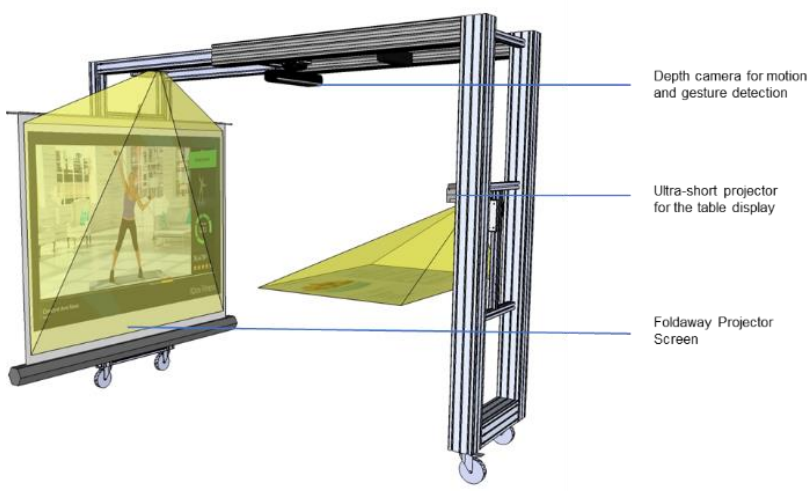

Figure 3. Prototypical version of the $\mathrm{PI}^{2} \mathrm{U}-$ SilverArc

\subsection{PI'U-MiniArc}

The $\mathrm{PI}^{2} \mathrm{U}-\mathrm{MiniArc}$ can be considered as a flexible and smaller variant of the $\mathrm{PI}^{2} \mathrm{U}-$ SilverArc, which is designed to assist in the training and moving of the elderly who are in hospital or live in smaller apartments. An ultrashort projector can project the user interface on its foldaway table or on a separate table as needed. In addition, a motion-sensing camera (Microsoft Kinect) is integrated to detect the user's gestures, enabling the interactive gaming function. There is another projector on top of the device that can project extra information onto a wall. This prototype is equipped with wheels and thus is mobile. Figure 4 and Figure 5 respectively demonstrate the preliminary design and the final prototypical version of the $\mathrm{PI}^{2} \mathrm{U}-\mathrm{Mini}$ Arc.

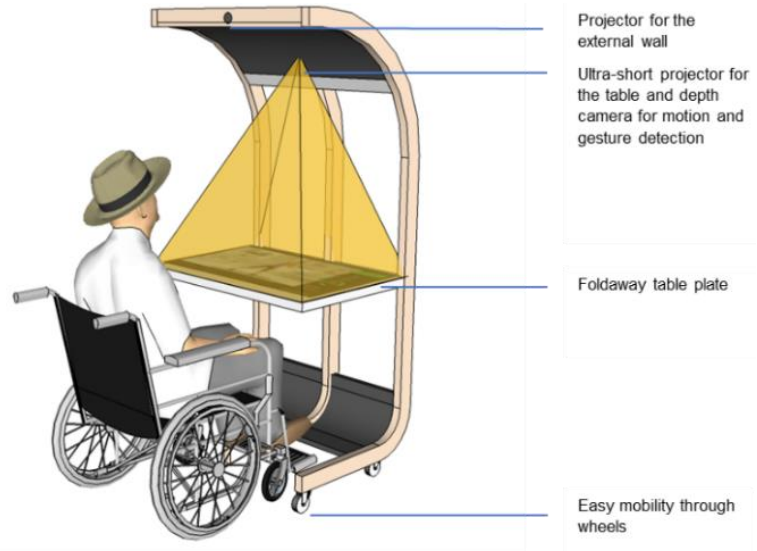

Figure 4. Preliminary design of the $\mathrm{PI}^{2} \mathrm{U}-$ MiniArc

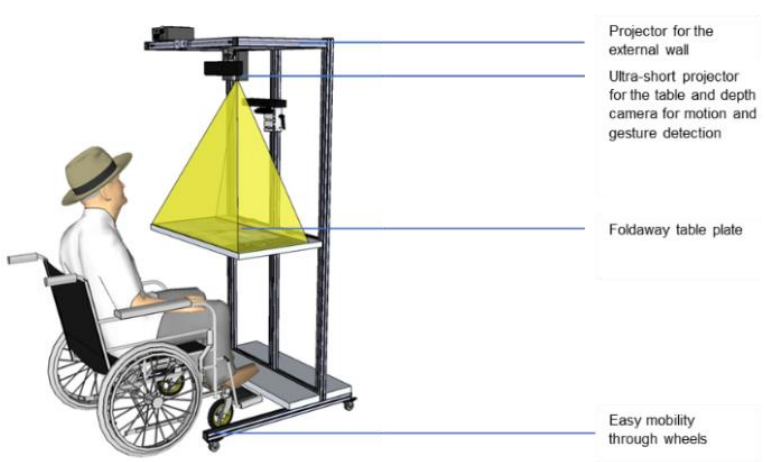

Figure 5. Prototypical version of the $\mathrm{PI}^{2} \mathrm{U}-$ MiniArc

\section{3 $\quad \mathbf{P I}^{2} \mathrm{U}$-Bed}

As shown in Figure 6, the first design of the PI² UBed resembles a normal bed for private use. A special feature is an arc-shaped frame that covers the entire length of the bed, which allows for an easy integration of sensors and technologies such as a thermal camera for breath detection and a projector for the bed. The height of the bed can be adjusted. On the one hand, this feature makes it possible for the caregivers to work at a height that is comfortable for their torsos. On the other hand, the lowest height of the bed facilitates the transfer of the patient from the bed to other functional units such as a wheelchair. The bed can be set to both a sitting and a vertical position. The sitting position allows the bed to support the patient and the nurse in many tasks such as eating, while the vertical position is especially apt for patients in an Intensive Care Unit (ICU) who must perform the transfer from lying to vertical position. The passive standing that is enabled by a standing frame aims to improve respiratory function and cardiovascular fitness, increase the levels of consciousness, functional 
independence, and psychological well-being, and reduce the risk for delirium and the adverse effects of immobility [5].

In order to adapt the bed system to each patient's needs, a modular docking system was integrated into the design. Modules providing additional functions such as a toilet, physical training, transfer, and mobility can dock at different positions in the frame of the bed and are symmetric and self-guided. For example, with the Leg-curl Training Module (see Figure 6), the patient can rest on their abdomen and use their legs to move the weight up and down. Training muscles is important to the performance of daily tasks.

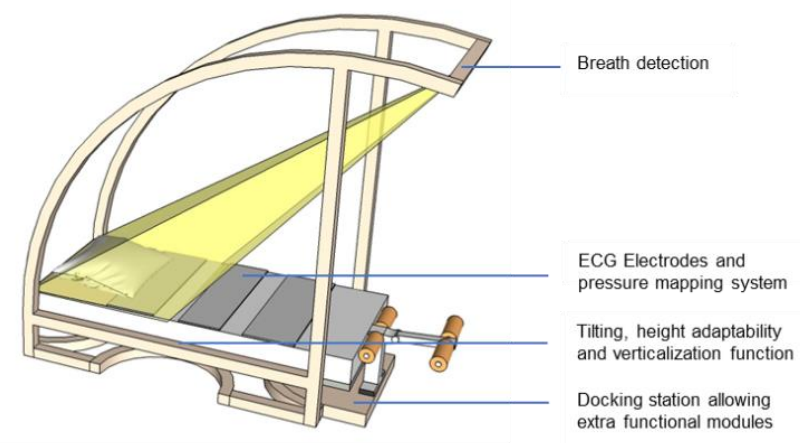

Figure 6. Preliminary design of the $\mathrm{PI}^{2} \mathrm{U}-\mathrm{Bed}$

To validate the concept of the $\mathrm{PI}^{2} \mathrm{U}-\mathrm{Bed}$, a prototype was planned and built. Figure 7 shows the final design of the $\mathrm{PI}^{2} \mathrm{U}$-Bed prototype that was manufactured.

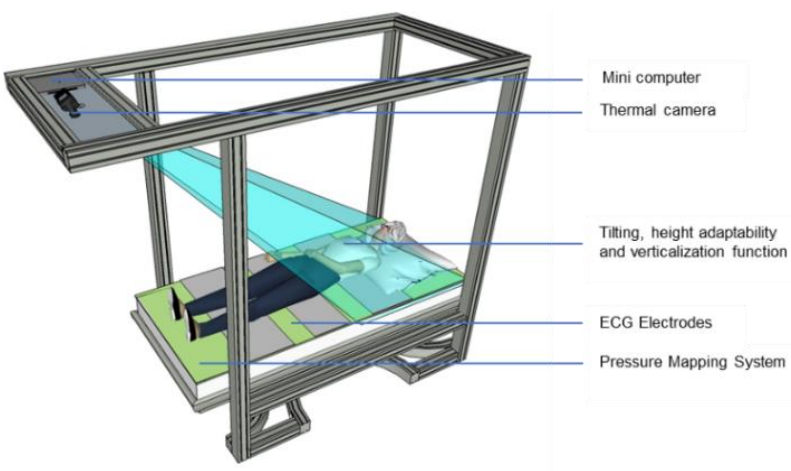

Figure 7. Prototypical version of the $\mathrm{PI}^{2} \mathrm{U}-\mathrm{Bed}$

\subsection{PI ${ }^{2} \mathrm{U}$-iStander}

The $\mathrm{PI}^{2} \mathrm{U}-\mathrm{i}$ Stander is designed to activate the physical and mental activity of people of old age whose daily activity level has reduced. It is used to prevent elderly people from falling and provides effective support for cognitive processes through the combination of physical and cognitive exercises. Due to its flexibility and reasonable size, the system can be easily deployed in spaces such as living rooms and bedrooms (see
Figure 8). The device is equipped with a mechanism to assist the elderly user to stand up and to perform movement exercises of the ankles, knees, and hip joints. A special corset and a seat ensure safety during the exercises that strengthen the back and abdominal muscles by lifting the legs. It also allows the user to maintain a safe, upright standing position and perform balance exercises as well as exercises where the upper body parts are activated using the ActiveLife gaming platform (see Figure 9) [6].

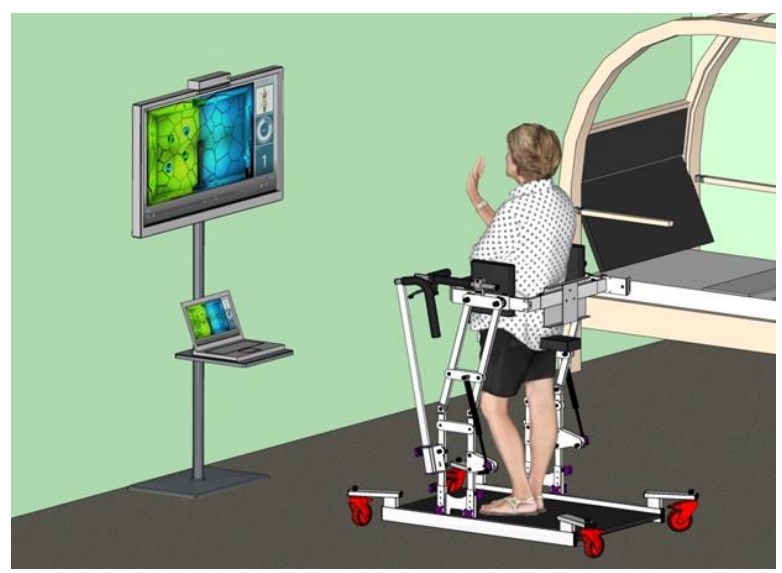

Figure 8. ActivLife system deployed in a bedroom environment
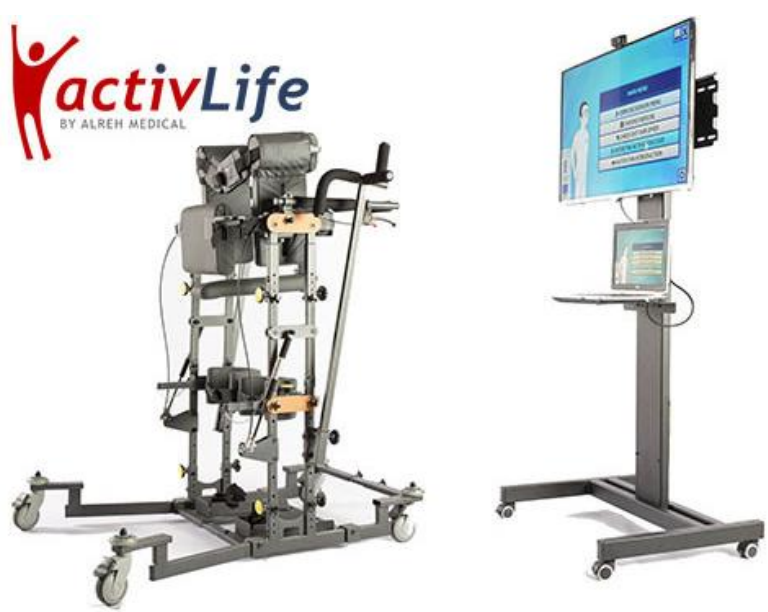

Figure 9. PI²U-iStander (Image: Alreh Medical)

\subsection{Major sensing, monitoring, and analysis activities using the $\mathrm{PI}^{2} \mathrm{Us}$}

As mentioned in the previous section, the project team in TP2 developed a series of Personalized Intelligent Interior Units (PI' $\left.{ }^{2} \mathrm{Us}\right)$, which are a special type of smart furniture that materialize the $\mathrm{REACH}$ concepts and functionality seamlessly into the different REACH use case settings. The $\mathrm{PI}^{2} \mathrm{Us}$ consist of the $\mathrm{PI}^{2} \mathrm{U}-$ SilverArc, $\mathrm{PI}^{2} \mathrm{U}-\mathrm{Mini} A r c, \mathrm{PI}^{2} \mathrm{U}-\mathrm{Bed}$, and $\mathrm{PI}^{2} \mathrm{U}-$ 
iStander. The $\mathrm{PI}^{2} \mathrm{U}$ prototypes are manufactured, currently deployed, and tested for major, sensing, monitoring, and analysis activities in REACH in the laboratory of the Chair of Building Realization and Robotics (BR2) at Technical University of Munich (TUM) (see Figure 10).

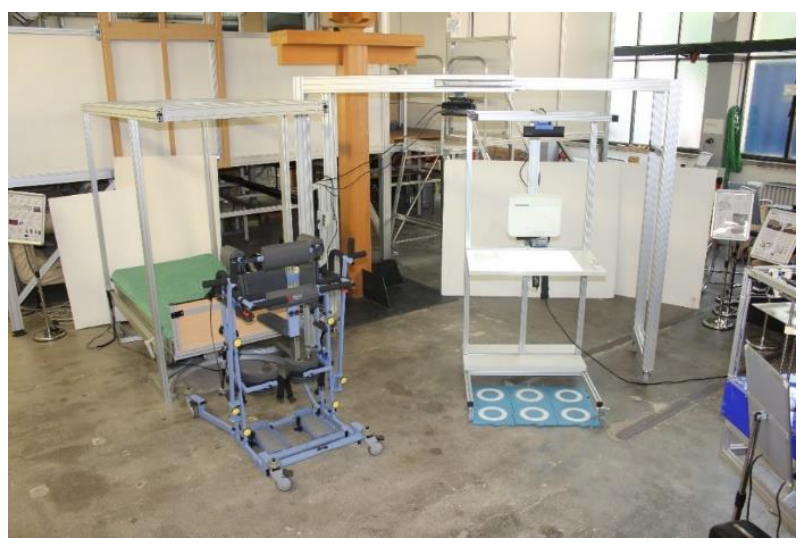

Figure 10. The prototypes of various $\mathrm{PI}^{2} \mathrm{Us}$ (e.g., $\mathrm{PI}^{2} \mathrm{U}-$ SilverArc, $\mathrm{PI}^{2} \mathrm{U}-\mathrm{Mini} A r c, \mathrm{PI}^{2} \mathrm{U}-\mathrm{Bed}$, and $\mathrm{PI}^{2} \mathrm{U}-\mathrm{i}$ Stander) deployed and tested in the laboratory

The electrocardiogram (ECG) sensors, which are embedded in the $\mathrm{PI}^{2} \mathrm{U}-\mathrm{Bed}$, will provide the medical staff with data regarding the patient's heart activity during the sleeping period. Additionally, this aspect can support the early detection aspect of the REACH project. Figure 11 presents the sensor integration on the $\mathrm{PI}^{2} \mathrm{U}$ Bed, which resulted in the ECG measurement signals in the second image (Figure 11 middle). In order to implement such sensors, flexible plastic material was used to improve patient comfort and measurement (Figure 11 right). The ECG implementation uses two electrodes to produce the ECG signal.
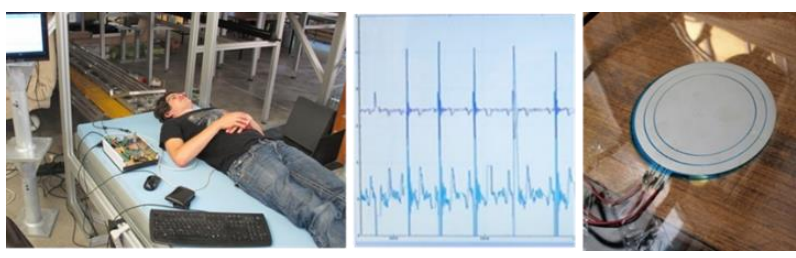

Figure 11. Testing of the ECG sensors on the $\mathrm{PI}^{2} \mathrm{U}-\mathrm{Bed}$

An important issue when considering an immobile patient's care is the prevention and management of pressure ulcers or decubitus. In order to implement the early detection and prevention aspects of REACH in the $\mathrm{PI}^{2} \mathrm{U}-\mathrm{Bed}$, it was decided to integrate a pressure-sensing mattress. This mattress monitors the peak pressure points of a person lying on the bed. Using this data, the REACH Engine can monitor peak pressure points and inform the care personnel for repositioning the patient before they develop decubitus. This sensor provides other functions as well. Using this sensor, a breath frequency monitor mainly for patients sleeping on their abdomens (see Figure 12) as well as a micro-mobility monitor are currently under implementation. Additionally, the project team is currently investigating the possibility of monitoring heart rate.

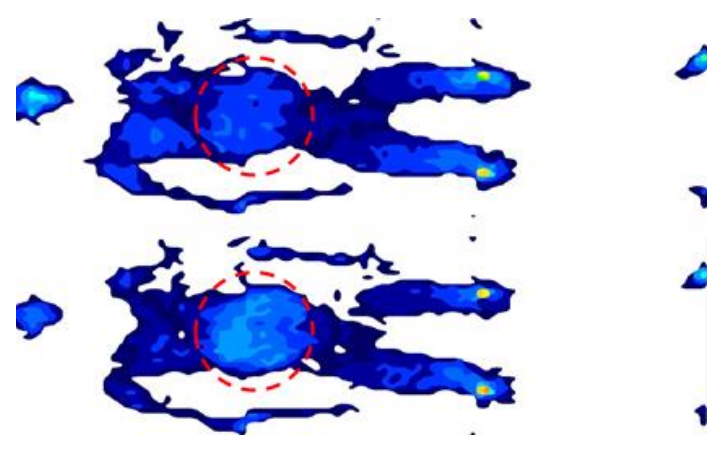

Figure 12. Pressure change on diaphragm when inhaling and exhaling (generated by the pressuresensing mattress)

The thermal camera targets two major objectives: 1) breath frequency monitoring over the nostrils during sleep on the back, and 2) body temperature detection/monitoring over the eyes (see Figure 13 and 14). In the first implementation of these modules in TUM's laboratory, the results showed the following: breath frequency monitoring is possible and implemented via the nostrils and that the body temperature monitoring is implemented (monitoring the body temperature via the eyes is, naturally, only possible before the patient goes to sleep). There is a small limitation regarding the implemented prototype; due to the resolution of the current thermal camera, it is only possible to monitor these factors in a range of approximately $50 \mathrm{~cm}$ distance from the patient's face. As a result, the thermal camera must either be mounted at the appropriate distance to the face, or the thermal camera must be replaced with a higher quality camera.

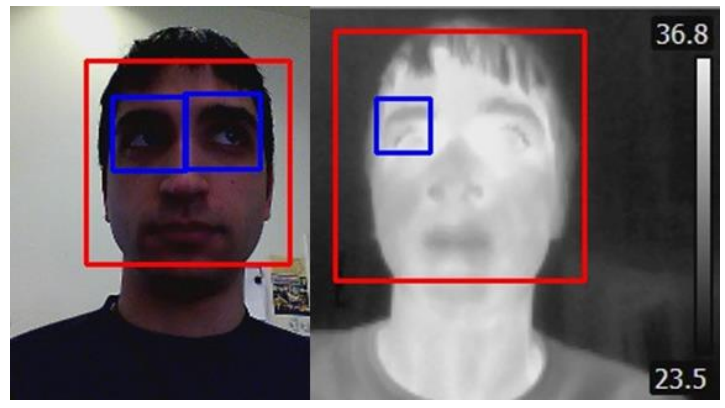

Figure 13. Eye following and body temperature measurement 


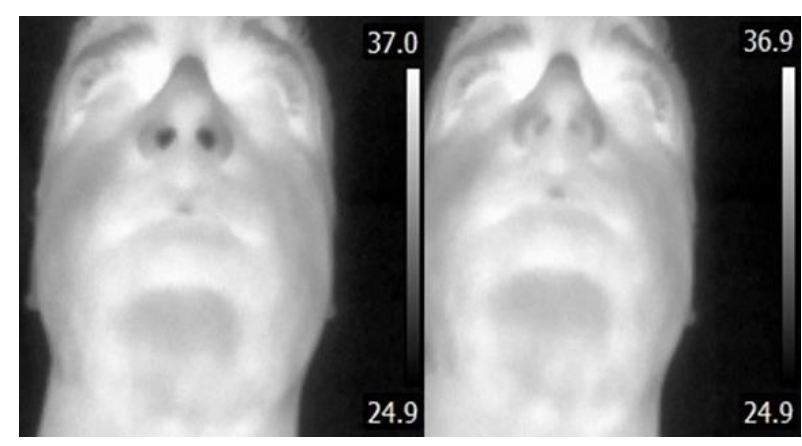

Figure 14. Respiration rate monitoring and body temperature measurement

The Kinect sensors, integrated into the $\mathrm{PI}^{2} \mathrm{U}$ MiniArc and $\mathrm{PI}^{2} \mathrm{U}-\mathrm{Sil}$ erArc, are used for gesture recognition by considering the hand motions from the user side (see Figure 15). The Kinect was programmed by using the standard libraries from Microsoft. Therefore, it is necessary to continue with Microsoft Windows as the operating system since the libraries of the Kinect are not compatible with other operating systems. The control program of the Kinect gesture recognition was programmed using visual studio and was developed separately from the Graphical User Interface (GUI). Furthermore, the software development of the mounted Kinect on the $\mathrm{PI}^{2} \mathrm{U}-$ MiniArc is finalized including the adjustability for recognizing gesture at two different distances (i.e., standing table surface and sitting table surface).

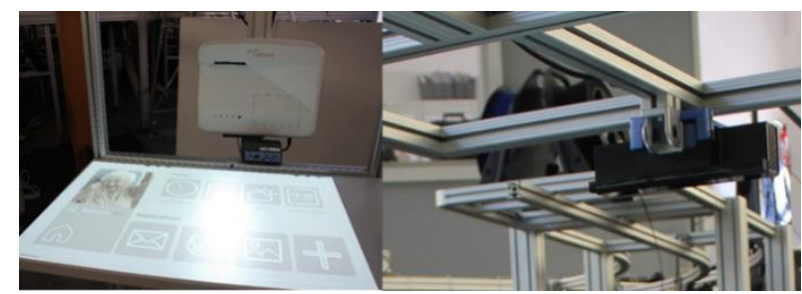

Figure 15. User interface and Kinect sensor in the $\mathrm{PI}^{2} \mathrm{U}-$ MiniArc

Designed and implemented as "battery-based" and "plug-and-play", the stand-up counting sensor is mounted on the Alreh Medical device in a specific way, so that the clinical certificate of the device will not be undermined (see Figure 16). This sensor counts the number of "stand-up" events and transfers this count to a local server via WiFi. In addition, this sensor is implemented with consideration of its exceptionally low power consumption. With the implemented battery, it will run for more than three months without the need for recharging the battery.

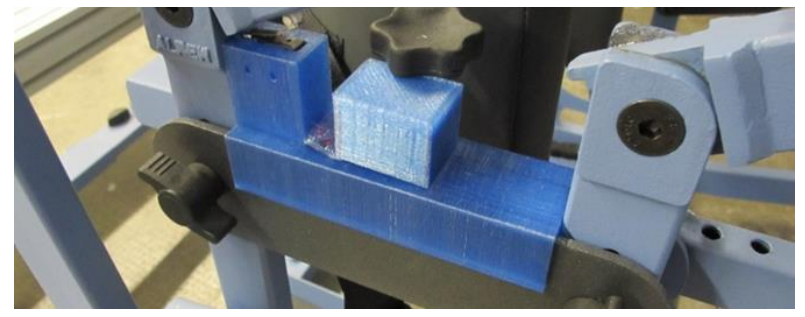

Figure 16. Stand-up counter embedded in the $\mathrm{PI}^{2} \mathrm{U}$-iStander

It is planned to implement an activity sensing system for the Alreh Medical device (see Figure 17). This activity sensing system will be fused with the current gaming interface of the Alreh Medical device in order to enable lower body interactions with the games. In the first trial, the electromyography (EMG) sensing was implemented and tested. EMG is an electrodiagnostic medicine technique for evaluating and recording the electrical activity produced by the muscles. This was planned to serve as the controller interface for the legs allowing the user to steer a training or rehabilitation game via gestures (e.g., by the Kinect sensor) and leg movements. After the initial implementations and tests in TUM's laboratory, the results showed immense amounts of noise when reading the EMG signal over the clothes. As a result, the project team planned to change the approach with the sensor electrodes and read the user activity from different sensors (e.g., touch sensors as previously mentioned). Afterwards, such inputs can be used to steer a training or rehabilitation game via foot gestures. Additionally, it will implement early detection, monitoring, and activation for the elderly.

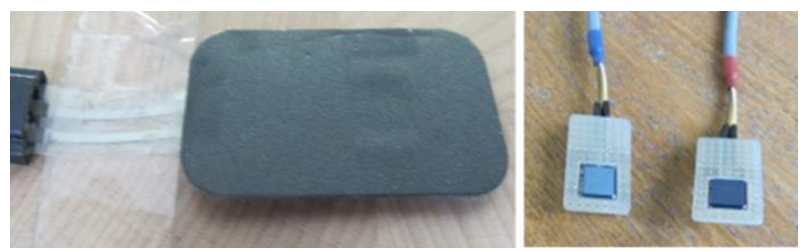

Figure 17. Activity monitoring sensors used in the $\mathrm{PI}^{2} \mathrm{U}-\mathrm{iStander}$

Currently, the overall testing of the $\mathrm{PI}^{2} \mathrm{Us}$ was successful. Based on the feedback from the testing, the $\mathrm{PI}^{2} \mathrm{Us}$ will be further revised and eventually resemble the proposed furniture design. After the early prototype testing of functionality and the first data collection, TUM proposed a design of a modularized apartment integrating all $\mathrm{PI}^{2} \mathrm{Us}$ and key technologies in REACH's four TPs to create a total interior living and care environment for elderly users. This design demonstrates the comprehensive REACH platform and can be adapted and implemented partially or entirely to different use case settings in an easy and rapid manner. 


\section{The REACH Apartment integration and simulation}

As mentioned in the previous section, the project team in TP2 developed a series of Personalized Intelligent Interior Units ( $\mathrm{PI}^{2} \mathrm{Us}$ ), which are a special type of smart furniture, that materialize the REACH concepts and functionality seamlessly into the different REACH use case settings. The $\mathrm{PI}^{2} \mathrm{Us}$ consist of $\mathrm{PI}^{2} \mathrm{U}-$ SilverArc, $\mathrm{PI}^{2} \mathrm{U}-\mathrm{Mini} A r c, \mathrm{PI}^{2} \mathrm{U}-\mathrm{Bed}$, and $\mathrm{PI}^{2} \mathrm{U}-\mathrm{i}$ Stander. Based on the current outcomes from four TPs, TUM designed a modularized apartment integrating all $\mathrm{PI}^{2} \mathrm{Us}$ and key technologies in REACH to create a complete interior living and care environment for elderly users (see Figure 18).

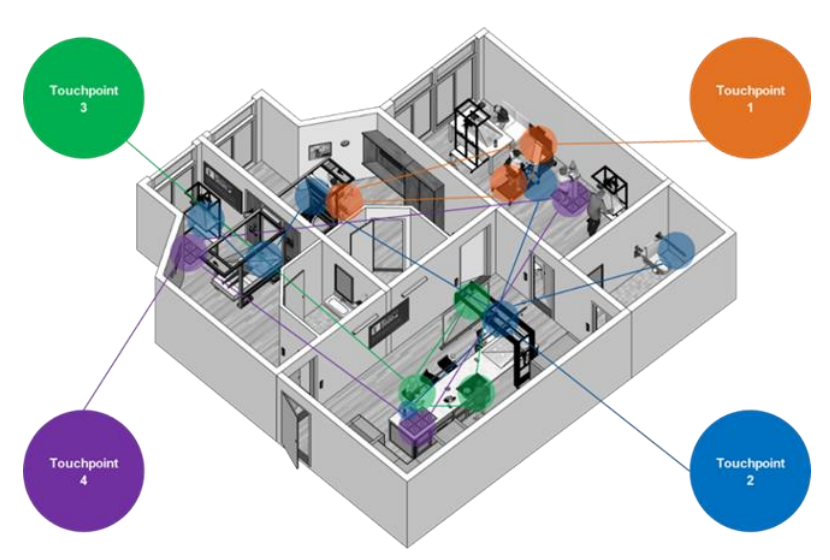

Figure 18. Technologies from four Touchpoints (TPs) distributed and integrated in the REACH apartment

Adopting barrier-free design principles, the apartment consists of a community kitchen, an activity room, and two patient rooms. In these living spaces, the Alreh Medical rehabilitation devices in TP1, smart furniture in TP2, socialized nutrition solutions in TP3, and gaming/training devices in TP4 are seamlessly integrated to create a comprehensive experience of the REACH platform for the users. Due to its modularity, parts of the apartment can be easily adapted and rapidly deployed in different REACH use case settings in four European countries, which will then help the REACH consortium execute a series of testing activities.

\subsection{The community kitchen}

The community kitchen serves as a key space for one of the most important daily activities - cooking and eating. It provides an ideal application space for technologies, particularly from TP3 (i.e., the "Socializing \& Nutritional Monitoring/Intervention" working cluster of the project). The elderly users of the community kitchen can cook and eat together with the help of state-of-the-art technologies. As shown in Figure 19, the $\mathrm{PI}^{2} \mathrm{U}-\mathrm{Silver} A r c$ (or alternatively $\mathrm{PI}^{2} \mathrm{U}-\mathrm{MiniArc}$ ) will project an interactive cooking table allowing the elderly to cook in a smart and interactive way. Furthermore, the elderly in the kitchen are encouraged to use a series of smartphone apps. In addition, healthy and customized food developed by Biozoon [7] will be provided to the elderly who use the kitchen. As a result, the elderly are expected to become more socially active and nutritionally healthy through using the community kitchen.

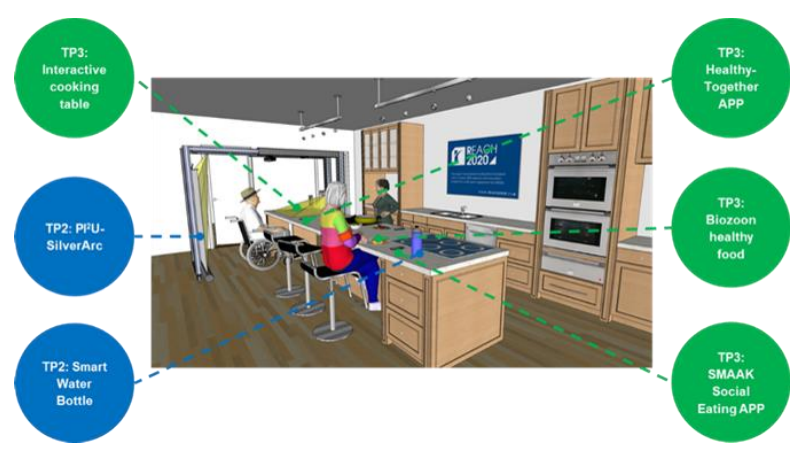

Figure 19. Simulation of the community kitchen

\subsection{The activity room}

One of the major research goals of the REACH project is to promote the activity level of elderly people. Therefore, the activity room provides a flexible space for the elderly to become more physically and mentally active. In this room, the ActivLife gaming platform and the $\mathrm{PI}^{2} \mathrm{U}$-iStander training device (developed by Alreh Medical), the $\mathrm{PI}^{2} \mathrm{U}-$ MiniArc in both table mode and mobile mode, and the Playware Moto Tiles (mototiles.com) will be implemented to provide the elderly with an active environment to improve their physical and mental health (see Figure 20).

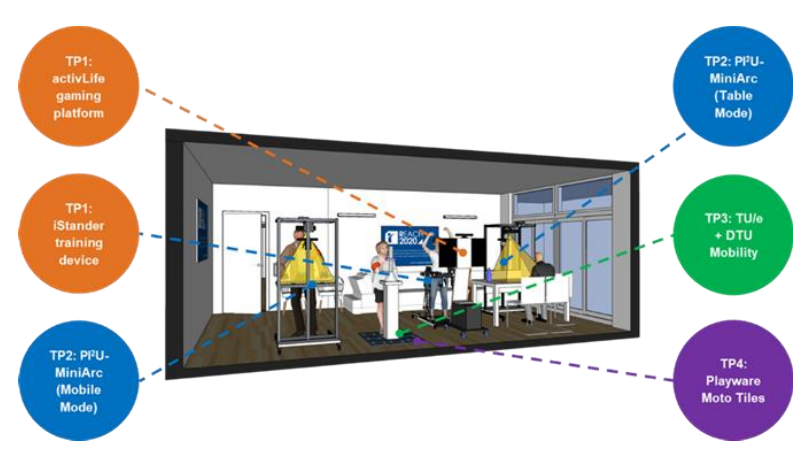

Figure 20. Simulation of the activity room

\subsection{The patient rooms}

In the patient room, the elderly can independently live, exercise, and play cognitive games using the $\mathrm{PI}^{2} \mathrm{U}-$ 
MiniArc. Meanwhile, when the patient is resting on the $\mathrm{PI}^{2} \mathrm{U}-\mathrm{Bed}$, the bed can monitor the user's body temperature and respiration rate by using a thermal camera, the body pressure can be measured using the Pressure Mattress, and ECG data can be monitored by using ECG sensors on the bed (see Figure 21).

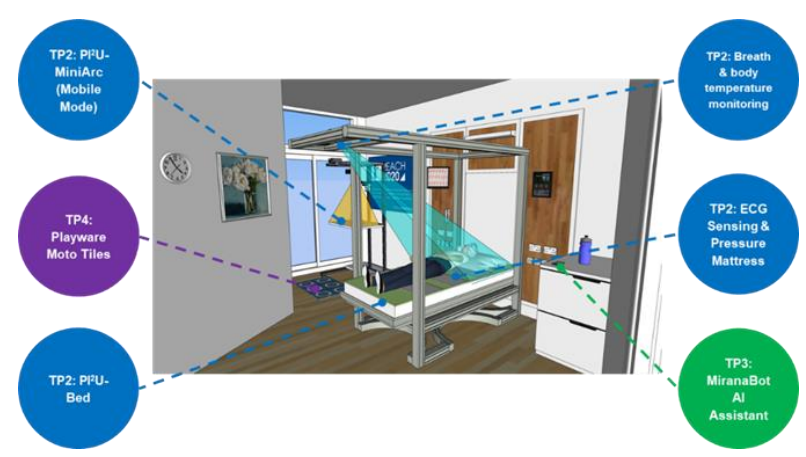

Figure 21. Simulation of the patient room type A

Figure 22 shows a variant of the patient room, in which the $\mathrm{PI}^{2} \mathrm{U}-\mathrm{Bed}$ rises to a vertical position, helping the user stand up easily and transfer into a $\mathrm{PI}^{2} \mathrm{U}-\mathrm{i}$ Stander with or without the help of a caregiver. The PI²UiStander serves as a training device for standing up as well as a mobility device to help the user move to other areas easily (e.g., bathroom, kitchen, activity room, etc.).

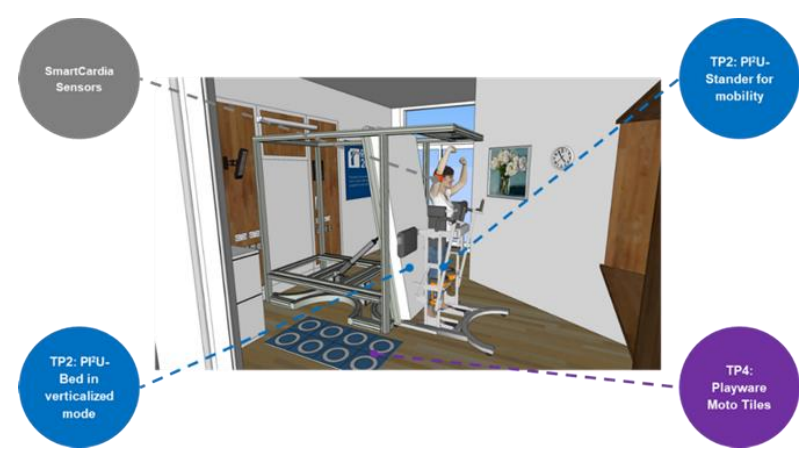

Figure 22. Simulation of the patient room type B

\section{Conclusion}

In this paper, the overall information of the REACH project as well as the detailed designs of the $\mathrm{PI}^{2} \mathrm{Us}$ and their prototypes are reported. The $\mathrm{PI}^{2} \mathrm{U}$-SilverArc has two interfaces that can be adapted to any large kitchen. Training programs and culinary recipes can be played on the interfaces. The $\mathrm{PI}^{2} \mathrm{U}$-MiniArc is a smaller and more mobile version of the $\mathrm{PI}^{2} \mathrm{U}-$ SilverArc. Both $\mathrm{PI}^{2} \mathrm{Us}$ aim to increase the social inclusion of their users. The $\mathrm{PI}^{2} \mathrm{U}-\mathrm{Bed}$ is a patient bed which can assist the patient and caregivers in various daily tasks. With the $\mathrm{PI}^{2} \mathrm{U}-$ iStander and the ActivLife gaming platform, the elderly can safely train their leg muscles without the risk of fall and train the upper body with ease and comfort.
Furthermore, the main testing, sensing, monitoring, and analysis activities using the $\mathrm{PI}^{2} \mathrm{U}$ prototypes are smoothly undertaken in TUM's laboratory. Based on the functionality validation of the $\mathrm{PI}^{2} \mathrm{Us}$, a comprehensive REACH apartment design integrating all $\mathrm{PI}^{2} \mathrm{Us}$ and technologies of REACH is proposed, which can later be adapted and implemented partially or entirely to different use case settings. As of writing this paper, the testing in TUM's laboratory is performed mainly with young healthy individuals. The usability and performance of these devices will be further validated by elderly testing persons, and the results will be revealed in subsequent experiments and research.

\section{Acknowledgements}

This project has received funding from the European Union's Horizon 2020 research and innovation programme under grant agreement No

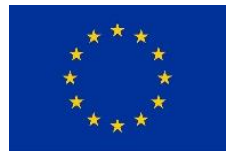
690425. The authors would like to thank all partners of the REACH consortium for their contributions to the presented work. Furthermore, the authors would like to express their gratitude to Mr. Andreas Bittner, Ms. Charlie Zhao, and Dr. Katharina Langosch for their support in this project.

\section{References}

[1] Espinoza, J. Europe's Failing Health. http://www.wsj.com/articles/SB100014240527487 04893604576200724221948728, Accessed: 03/01/2019.

[2] Lipszyc, B., Sail, E., and Xavier, A. Long-term care: need, use and expenditure in the EU-27. Directorate General Economic and Monetary Affairs, European Commission, 2012.

[3] McPhee, J. S., French, D. P., Jackson, D., Nazroo, J., Pendleton, N., \& Degens, H. Physical activity in older age: perspectives for healthy ageing and frailty. Biogerontology, 17(3): 567-80, 2016.

[4] Bock, T. REACH: Responsive Engagement of the Elderly promoting Activity and Customized Healthcare. Gerontechnology, 16(3): 125-128, 2017.

[5] Stiller, K., \& Phillips, A. Safety aspects of mobilising acutely ill patients. Physiotherapy Theory and Practice, 19: 239-257, 2003.

[6] Kozak, D., Burgermeister, S., De Chassey, J. D. B., Naefb, A., Maringue, A., \& Dietrich, D. A functionality, safety and validity study of innovative REACH devices. Gerontechnology, 16(3): 181-188, 2017.

[7] Biozoon. On-line: https://biozoon.de/en/, Accessed: 03.01.2019. 